\title{
Steps in the evolution of heteromorphic sex chromosomes
}

\author{
D Charlesworth ${ }^{1}$, B Charlesworth ${ }^{1}$ and G Marais ${ }^{1,2}$ \\ ${ }^{1}$ Institute of Evolutionary Biology, School of Biological Sciences, University of Edinburgh, King's Buildings, West Mains Road, \\ Edinburgh, UK; ${ }^{2}$ Bioinformatics and Evolutionary Genomics, UMR CNRS 5558, Gregor Mendel Building, University of Lyon, \\ Villeurbanne, France
}

\begin{abstract}
We review some recently published results on sex chromosomes in a diversity of species. We focus on several fish and some plants whose sex chromosomes appear to be 'young', as only parts of the chromosome are nonrecombining, while the rest is pseudoautosomal. However, the age of these systems is not yet very clear. Even without knowing what proportions of their genes are genetically degenerate, these cases are of great interest, as they may offer opportunities to study in detail how sex chromosomes evolve. In particular,
\end{abstract}

we review evidence that recombination suppression occurs progressively in evolutionarily independent cases, suggesting that selection drives loss of recombination over increasingly large regions. We discuss how selection during the period when a chromosome is adapting to its role as a $\mathrm{Y}$ chromosome might drive such a process.

Heredity (2005) 95, 118-128. doi:10.1038/sj.hdy.6800697; published online 25 May 2005

Keywords: genetic degeneration; repetitive sequence; pseudoautosomal region; recombination suppression; evolutionary strata

\section{Introduction}

The fundamentals of how and why sex chromosomes evolve have been reviewed many times (Bull, 1983; Charlesworth, 1996, 2002). It is well understood that the idea that sex-determining genes should not recombine (Nei, 1969) can explain the lack of recombination between $\mathrm{X}$ and $\mathrm{Y}$ chromosomes of animals such as Drosophila and mammals with male heterogamety (or $\mathrm{Z}$ and $\mathrm{W}$ in taxa with female heterogamety such as birds and Lepidoptera; Bull, 1983). It is also well established that such nonrecombining $\mathrm{Y}$ or $\mathrm{W}$ chromosomes have lost many genes that are carried on the ancestrally homologous $\mathrm{X}$ or $\mathrm{Z}$ chromosomes (genetic degeneration; Bull, 1983; Charlesworth and Charlesworth, 2000). In mammals, only a few X-linked genes have homologues on the $\mathrm{Y}$ chromosome, and several Y-linked genes are known that do not have homology to X-linked genes and have presumably been translocated to the $\mathrm{Y}$ from elsewhere (Lahn et al, 2001); in D. melanogaster, all known Y-linked genes are in this latter category (Carvalho, 2002). The $X$ and $Y$ (or $Z$ and $W$ ) chromosomes are often morphologically distinguishable (heteromorphic), with the $\mathrm{Y}$ or $\mathrm{W}$ being largely heterochromatic and containing a high proportion of repetitive DNA (Bull, 1983).

Our existing theories of the early stages of sex chromosome evolution show how the lack of recombina-

Correspondence: D Charlesworth, Ashworth Lab., Institute of Evolutionary Biology, School of Biological Sciences, University of Edinburgh, King's Buildings, W. Mains Rd., Edinburgh EH9 3JT, UK.

E-mail: Deborah.Charlesworth@ed.ac.uk

Received 30 November 2004; accepted 24 March 2005; published online 25 May 2005 tion between the sex chromosomes may have arisen (Charlesworth and Charlesworth, 1978), and how this can lead to genetic degeneration of the heterozygous sex chromosome (reviewed in Charlesworth and Charlesworth, 2000). Indeed, cessation of recombination between large parts or all of the sex chromosomes is the ultimate cause of the degeneration of $\mathrm{Y}$ or $\mathrm{W}$ chromosomes. In the first place, isolation caused by the cessation of recombination is a necessary condition for genetic divergence between the two sex chromosomes to begin. Second, the lack of recombination among most genes carried on $\mathrm{Y}$ or $\mathrm{W}$ chromosomes reduces the ability of selection to fix favourable mutations (Orr and Kim, 1998) and to prevent the fixation of deleterious ones, due to Hill-Robertson interference among completely linked loci (hitch-hiking processes reviewed in Charlesworth and Charlesworth, 2000). The sheltering of recessive or partly recessive Y-linked deleterious mutations from selection due to their permanent heterozygosity may also contribute (Nei, 1970) but seems unlikely to be the main force involved (Charlesworth and Charlesworth, 2000). In addition to these models, which predict gradual loss of the level of adaptation of genes on the $\mathrm{Y}$ or $\mathrm{W}$ chromosomes, there are several well-characterised population genetic processes that cause a build-up of both tandem arrays of satellite-type DNA sequences and transposable elements in genomic regions where crossing over is reduced or absent (Charlesworth et al, 1994), without necessarily impairing fitness.

The properties of lack of recombination and genetic degeneration are so closely connected in our ideas of how sex chromosomes evolve that they define our current concept of sex chromosomes, but it should be remembered that other heteromorphic chromosome 
systems with suppressed recombination show some striking similarities to sex chromosomes. The Chlamydomonas mating-type locus (Ferris et al, 2002) and incompatibility locus regions of some fungi with systems with two incompatibility types (Fraser and Heitman, 2004) include large nonrecombining genome regions that differ in size, gene content and representation of transposable elements. The heteromorphic sex chromosomes of bryophytes, which are primarily haploid (Bull, 1983; Ishizaki et al, 2002), represent a similar situation, although it is not yet known whether these homologous chromosome pairs show genetic degeneration. These haploid systems support the conclusion above that lack of recombination is sufficient for gene loss and transposable element accumulation. Plant self-incompatibility loci may be similar (Mather and Winton, 1941; Kurian and Richards, 1997; Stone, 2004), although the sizes of nonrecombining regions, and even their existence, are not yet established (Takebayashi et al, 2004).

As sex chromosomes are a special case of a more general phenomenon, it has been suggested that all these different cases should be called sex chromosomes (Fraser and Heitman, 2004). Here, we will restrict the term to chromosomes that carry the genes controlling male or female development, defining the male-female dichotomy in terms of gamete size differences (Maynard Smith, 1978), which are absent in other systems. Another difference is that in diploid species one sex chromosome (the $\mathrm{X}$ or $\mathrm{Z}$ ) is regularly present in two copies in one sex and undergoes crossing over, whereas the sex-specific chromosome or region is always heterozygous and nonrecombining. Most mating-type loci have no such asymmetry, all alleles generally being heterozygous in the diploid parts of the life cycle; in plants with the distylous form of heterostyly, however, one incompatibility type is heterozygous while the other is homozygous, similarly to sex chromosomes (Barrett, 1992). The permanent heterozygosity of evolving $\mathrm{Y}$ or $\mathrm{W}$ chromosomes clearly favours the erosion of genes on these chromosomes, since the active counterparts on the $\mathrm{X}$ or $\mathrm{Z}$ chromosomes can maintain their functions, especially if dosage compensation evolves (Charlesworth, 1978).

The use of molecular markers in mapping experiments to identify regions involved in sex determination in species without heteromorphic sex chromosomes, and the molecular genetic characterisation of sex-specific loci, have revolutionised our ability to study sex chromosome systems outside the standard model organisms. We will show how these new approaches have advanced our understanding of the evolution of sex chromosomes. We will not review the theory of $\mathrm{X} / \mathrm{Y}$ chromosome evolution, or describe classical sex chromosomes, but will describe new information from taxonomically diverse species, in many of which the sex-determining genes are not located on classical heteromorphic sex chromosomes, but within much smaller nonrecombining genomic regions.

\section{Types of sex chromosomes}

Studies of a wide diversity of animal and plant species have found differing degrees of genetic and structural differentiation between the two sex chromosomes (Bull, 1983). The situation closest to the theoretical models of the origins of sex chromosomes should be found in recently evolved sex chromosomes. The most common ancestral state in plants is probably hermaphroditism, although dioecy can also evolve from monoecy (with developmentally well differentiated male and female flowers already having evolved). Animal genetic sexdetermining systems may, however, often have evolved from environmental sex determination, again with established male and female organs and physiology (Janzen and Krenz, 2004).

According to our theoretical understanding, plant or animal sex chromosomes that evolved recently from any of these ancestral states should contain a region with a cluster of sex-determining genes that are closely enough linked to allow separate sexes to evolve (Charlesworth and Charlesworth, 1978). The required degree of linkage depends on the effects of the genes involved. For instance, a female suppressor can evolve in a gynodioecious population, despite lowering the reproductive fitness of females, provided that its allele on the proto$\mathrm{Y}$ is linked to the locus causing male sterility in females, which defines a proto- $X$ chromosome (Figure 1). The linkage must be tight if the female-suppressor strongly reduces female fertility, but less tight linkage is necessary if the effect is milder. The presence of two such genes in this chromosome region then induces a selection pressure for reduced recombination in this part of the chromosome pair (a single sex-determining locus will not select for loss of recombination). It is often stated that sex chromosome evolution starts when one member of a chromosome pair acquires a sex-determination function (Carvalho, 2002), but this leaves out an important part of the evolution of genetic sex determination: two separate mutations, in order to produce genetically distinct females and males.

Recombination should thus initially be suppressed only in the sex-determining region. However, many sex chromosome pairs have suppressed recombination extending over much of the $\mathrm{Y}$, except for a small 'pseudoautosomal' region or regions (PAR) to which pairing and recombination are restricted. Since one crossover per chromosome arm is often required for disjunction, crossover rates are likely to be extremely high in these regions (Perry and Ashworth, 1999). Studies of young sex chromosomes are important for testing the model for the early stages, while data on older systems help test ideas about what causes suppressed recombination over more extensive genomic regions.

\section{What causes suppressed recombination?}

An interesting idea about why recombination suppression expands to include much of the $\mathrm{Y}$ or $\mathrm{W}$ chromosomes is that it is a consequence of the action of selection on other genes, which interact with the primary sexdetermining genes (Fisher, 1931; Bull, 1983; Rice, 1987b, 1996); we will discuss the $Y$, but similar considerations apply to W-linked genes. Such interactions involve alleles with good effects in one sex, but which reduce the fitness of the other sex (sexually antagonistic genes), creating genetic conflicts similar to those during the initial evolution of separate sexes. Mutations whose expression is sex limited can also spread, but this will probably apply to few mutations in young dioecious species. For many genes, therefore, their spread in dioecious populations is constrained by linkage, 
(1) Evolution of sex-determining region with male-sterility and female-sterility alleles at linked loci leads to a small sex-determining region on an ordinary chromosome

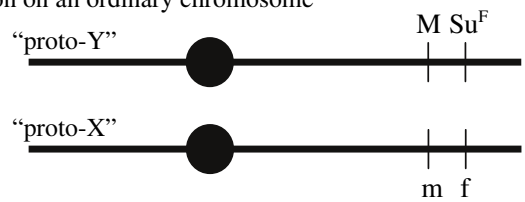

(2) Evolution of reduced recombination between these genes (and perhaps around them) Proto-Y
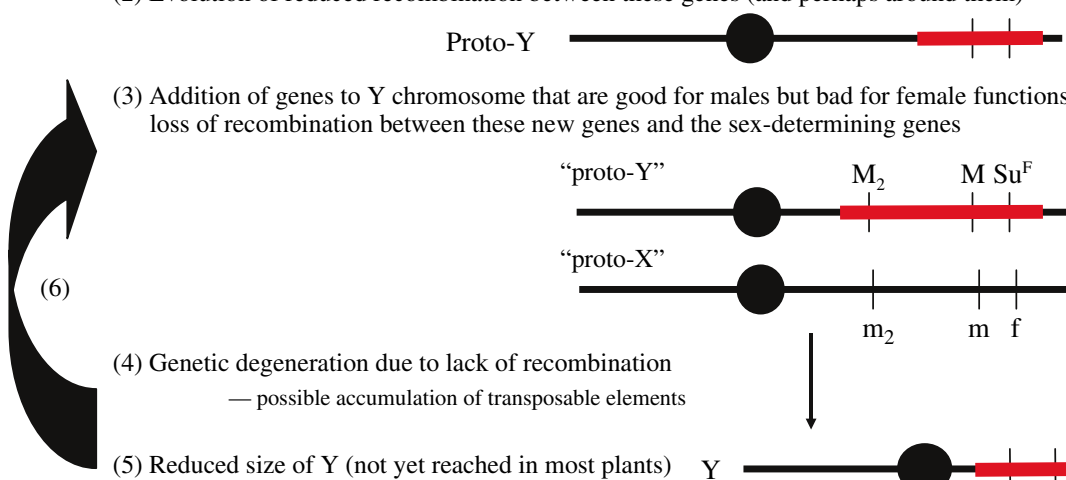

(3) Addition of genes to $\mathrm{Y}$ chromosome that are good for males but bad for female functions, and loss of recombination between these new genes and the sex-determining genes

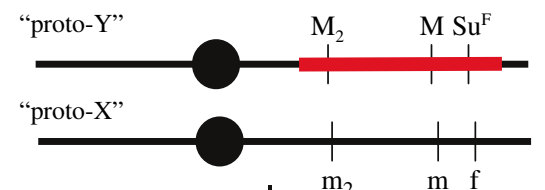

(4) Genetic degeneration due to lack of recombination — possible accumulation of transposable elements

(5) Reduced size of Y (not yet reached in most plants)
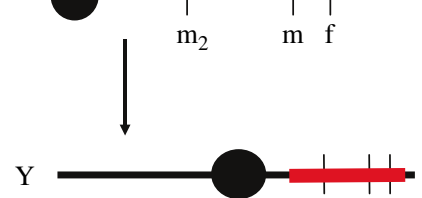

Figure 1 Stages in sex chromosome evolution. The figure shows how proto-sex chromosomes, carrying just two sex-determining genes causing male and female sterility on the proto- $X$ and proto- $Y$, respectively, may evolve by adding further genes whose effects interact with sex, leading to evolution of suppressed recombination over a wider genome region, followed by genetic degeneration and loss of nonessential parts of the Y chromosome. The simplest model involves an initial sequence of two genetic steps (part 1 of the figure) to change from a population of hermaphrodites to one with males and females (see text). One locus has initial state $M$, which undergoes the mutation $M \rightarrow m$, where $\mathrm{m}$ is a recessive male-sterility allele causing the appearance of females, and the initial allele $\mathrm{M}$ remains unchanged on the chromosome that later evolves to become the $\mathrm{Y}$ (the proto- $\mathrm{Y}$ ). The second locus undergoes the change $\mathrm{f} \rightarrow \mathrm{Su}^{\mathrm{F}}$, where $\mathrm{Su}^{\mathrm{F}}$ is a dominant female-suppressing allele changing hermaphrodites into males. More than two changes are also possible. In the primitive sex-determining region, selection favours low recombination between the proto-Y chromosome and its homologue. Part 2 of the figure shows this region in red after recombination with the proto-X has been suppressed and the region has thus become male specific. Further genetic changes may also occur on the sex chromosomes, such as the change of $\mathrm{m}_{2}$ to a male-promoting allele, $\mathrm{M}_{2}$. This addition of male-promoting genes leads to selection for suppressed recombination in a wider region (see text). Genetic degeneration of the male-specific region may then occur as outlined in the text, finally leading to loss of Y chromosome DNA.

similarly to the conditions just outlined for the primary sex-determining genes, so that fairly close linkage to the sex-determining loci on the proto- $Y$ chromosome is probably often necessary (Fisher, 1931). The Y chromosome is thus expected to accumulate genes beneficial for male functions, confining them to males (Rice, 1987a), and male attractiveness genes in guppies are indeed often partially Y-linked (Lindholm and Breden, 2002). Once such genes have accumulated, there is a selective advantage to suppressing crossing over between them and the sex-determining regions of the proto-sex chromosomes. As shown in Figure 1, at least two evolutionary factors are thus expected to lead to recombination suppression between sex chromosomes: (i) the presence of sex-determining genes that should not recombine, and (ii) the evolution of Y-linked genes that benefit male but not female functions, again causing selection against recombinants.

Two kinds of change might affect recombination between the evolving sex chromosomes. One possibility is a gradual reduction of crossover frequencies, due to the spread of genetic modifiers of recombination rates (Brooks, 1988). The second possibility involves chromosome rearrangements such as inversions, and these could also cause $X-Y$ chromosome heteromorphism. As few as two interacting genes can drive selection for reduced recombination via either process. Inversions on either the proto-Y or proto- $X$ may be favoured (Charlesworth and
Hartl, 1978), and may affect crossing over in a large part of the sex chromosomes, involving genome regions containing many genes, most of them with no role in sex determination. Where no rearrangements can be detected cytologically, we may infer that gradual change is likely. We will consider evidence bearing on the possibility of inversions below.

Translocations or centric fusions involving an autosome and a sex chromosome could also create linkage between sexually antagonistic genes and the sex chromosomes, and hence be favoured by natural selection (Charlesworth and Charlesworth, 1980). In addition to the creation of linkage between genes that were formerly on separate chromosomes, such rearrangements often reduce or suppress crossing over in the regions around the breakpoints in heterozygotes with the standard arrangement (Ashburner, 1989; Davisson and Akeson, 1993; Vieira et al, 2003). Chromosomes newly subjected to a sex chromosomal inheritance pattern in this way are called 'neo-sex chromosomes'. A classic example is the Drosophila miranda Y-autosome fusion, which involves the homologue of the third chromosome of its close relative $D$. pseudoobscura (Steinemann and Steinemann, 1998). In this case, the neo- $Y$ would immediately have stopped recombining due to the absence of crossing over in male Drosophila. In D. americana, a relative of $D$. virilis, an $\mathrm{X}$-autosome fusion is either fixed or polymorphic in some populations (McAllister, 2002). Here, the neo- $Y$ is 
not physically attached to the $\mathrm{Y}$, but cosegregates with the true $Y$ from the $X$ and the neo- $X$ in males.

Neo-sex chromosome systems are known in many plants and animals, including $X Y_{1} Y_{2}$ in the plant Rumex acetosa (Rejón et al, 1994), and multiple sex chromosomes due to translocations in African misteltoes (Barlow and Wiens, 1976), termites (Syren and Luykx, 1977), African pygmy mice (Veyrunes et al, 2004) and monotremes (Rens et al, 2004). As we shall describe below, the sex chromosomes of eutherian mammals represent an ancient case of neo-sex chromosomes, established after the divergence of eutherians and marsupials (Waters et al, 2001). Several additions to the mammalian sex chromosomes are inferred, including the recent addition of a second PAR2. Genes in both the present-day PARs are autosomal in marsupials, and are on different marsupial chromosomes (Charchar et al, 2003).

Finally, sex chromosome systems, once established, have been modified in various ways that have no direct consequences for recombination frequencies. Transpositions of autosomal genes have occurred onto established Y chromosomes in mammals (Skaletsky et al, 2003). All genes known on the $D$. melanogaster $\mathrm{Y}$ appear to be male function genes without $X$ chromosome counterparts, which were probably acquired from the autosomes (reviewed in Carvalho, 2002). The Y's lack of evident homology with the $X$, beyond pairing and carrying rDNA, has led to suggestions that the D. melanogaster $\mathrm{Y}$ is not a degenerated $X$ at all. The $Y$ chromosome of some insects may have evolved from a pre-existing, already nonrecombining, heterochromatic B chromosome (Nokkala et al, 2003), and such an origin could potentially account for the situation in D. melanogaster (Carvalho, 2002). This, however, seems speculative, and there is no direct evidence. Complete loss of functional genes from $Y$ chromosomes can readily account for absence of $Y-X$ homology. Degeneration of an X-homologous neo-Y of $D$. miranda is described below, and the ancestral Y chromosome has degenerated completely in D. pseudoobscura, after a fusion with an autosome created a neo- $X$, whose homologue probably became a new $Y$, which is now also genetically degenerated (Carvalho and Clark, 2005).

Another possible type of change is the emergence of a new sex-determining gene, epistatic to the previous sex determiner, so that the original genes no longer control sex development, as in the fly Musca domestica (Shearman, 2002), and probably also in the medaka fish, Oryzias latipes (see below). The sex-determining region of the fly, Megaselia scalaris, appears to transpose between chromosomes, creating new potential sex chromosomes (Traut and Willhoeft, 1990; Willhoeft and Traut, 1990; Traut and Wollert, 1998). By this type of process, a single locus takes control of sex determination, that is, derived states with a single sex-determining gene can evolve. Single locus sex determination, such as that of mammals, with a single Y-linked gene, SRY (Lahn et al, 2001), controlling sex, might also be able to evolve directly, but no detailed model has yet been worked out to show how this could occur.

Based on the ideas outlined above, single-gene sex determination situations should not select for reduced recombination in the new sex-determining region; such a selection pressure could, however, emerge as further genes interacting with the primary sex-determining genes evolve (Rice, 1996). New sex-determining regions of this kind should therefore allow tests of the theory that interactions between sexually antagonistic genes with sex-determining genes are important in the evolution of suppressed crossing over between the sex chromosomes.

\section{Gradual suppression of recombination and the role of inversions}

There is now evidence from several taxa (mammal, birds and plants) for more than a single event of cessation of recombination between $\mathrm{X}$ and $\mathrm{Y}$ chromosomes. The extent of DNA sequence divergence between homologous X/Y gene pairs of a single species has been found to vary greatly among genes, suggesting that different regions of the sex chromosomes have been nonrecombining for very different amounts of time, given that sequence divergence begins once crossing over between $\mathrm{X}$ and $\mathrm{Y}$ stops. Moreover, in these cases, divergence levels (called 'evolutionary strata' by Lahn and Page, 1999) show a clear pattern in relation to the $X$ chromosome genetic map; for loci that are still present on the $Y$, comparisons of $\mathrm{Y}$ and $\mathrm{X}$ homologues shows that the sequences of genes closest to the PAR have least synonymous site divergence, while loci further away belong to the older, more diverged sex chromosome region (Lahn and Page, 1999; Skaletsky et al, 2003; Lawson-Handley et al, 2004; Nicolas et al, 2005). A schematic diagram of such strata is shown in Figure 2. In the human $X$, there appear to be at least four strata (Lahn and Page, 1999; Ross et al, 2005). The human amelogenin gene, which spans the boundary of strata 3 and 4 , shows this effect within this single locus. Synonymous divergence between the AmelX and Y copies changes from $30 \%$ in the older $5^{\prime}$ region (in stratum 3) to $10 \%$ in the $3^{\prime}$ region (in stratum 4 ); in a multispecies study, the same pattern was found in other mammalian orders (Iwase et al, 2003). This pattern demands an explanation.

Was recombination suppression in mammals a discontinuous process (due to successive inversions or translocations), or did it evolve gradually? Gene order differs between the $X$ and $Y$ chromosomes, whereas $X$ chromosomes are remarkably homosequential between different mammals, suggesting $\mathrm{Y}$ rearrangements (Skaletsky et al, 2003). In humans, the finding of evolutionary strata, without any evident relationship between genes' positions on the $\mathrm{Y}$ chromosome and synonymous divergence between $X$ and $Y$ copies, prompted the suggestion that four inversions have occurred on the mammalian $\mathrm{Y}$.

There is some support for inversions. The XG gene, which spans the current human PAR boundary, has a truncated $Y$ copy, perhaps caused by an inversion (see references in Iwase et al, 2003). However, inversions are probably not the complete explanation for the lack of $X_{-}$ $Y$ crossing over. Translocations onto the $Y$ have certainly occurred; as already mentioned, the eutherian sex chromosomes are ancient neo-sex chromosomes, which partially explains the divergence differences. The oldest strata, 1 and 2, include genes that are also sex-linked in marsupials, whereas genes in eutherian mammals' strata 3 and 4 are autosomal in marsupials (Waters et al, 2001). The boundaries between strata, especially 3 and 4 , are not as clear as would be expected if the strata were created by inversions, and no evidence explicitly links 


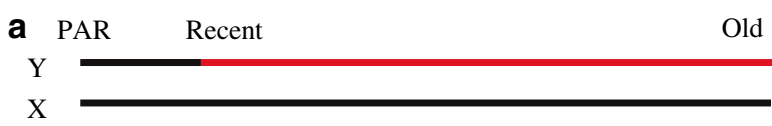

b

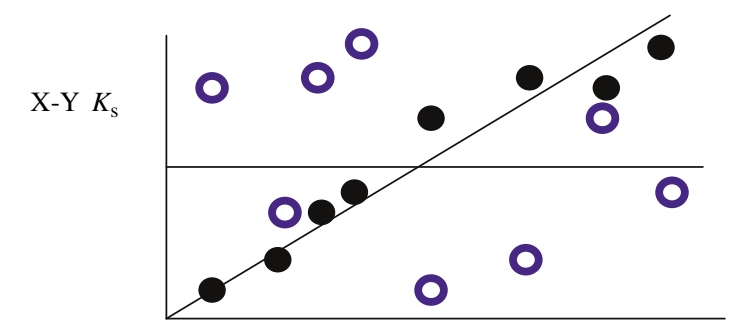

C

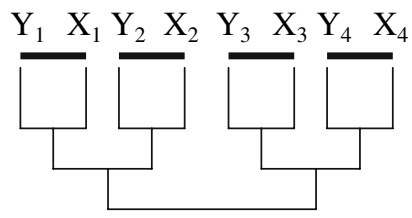

Genes in which recombination stopped after split of different taxa (indicated by bars)
Plotted against

location of X-linked

genes

Plotted against

location of Y-linked

genes
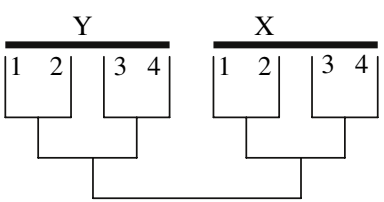

Genes that stopped recombining before taxa split

Figure 2 Schematic diagram to illustrate the behaviour of strata in sex chromosomes. (a) Diagram of a sex chromosome pair, showing the male-specific region, with 'old' regions where $X-Y$ recombination ceased long ago, and regions adjacent to the PAR, in which the $X$ and $Y$ recombined more recently; for simplicity, no rearrangements of the $\mathrm{Y}$ chromosome are shown. (b) Synonymous site divergence values between orthologous X-and Y-linked genes (the $y$-axis) are plotted against the locations of these genes on the X chromosome (the $x$-axis). (For actual mammalian data see Figures 7 and 8 of Skaletsky et al, 2003.) (c) The different phylogenetic patterns that would be observed for a gene in the old (right) and the new (left) regions of the sex chromosomes. Four hypothetical species are illustrated, and the tips of the gene trees are labelled with the species numbers. (For an example from birds, see Figures 2, 3 and 5 of Lawson-Handley et al, 2004.)

the strata with the history of rearrangements of the sex chromosomes. Moreover, the Y copy of the amelogenin gene at the boundary of strata 3 and 4 is not truncated, as would be expected if stratum 4 was formed by an inversion (Iwase et al, 2003). Finally, gene conversion can continue between the $\mathrm{X}$ and $\mathrm{Y}$ chromosomes after they have lost the ability to cross over (Pecon-Slattery et al, 2000; Marais and Galtier, 2003; Rozen et al, 2003), so variation in the rate of gene conversion could contribute to the variation in extent of $X / Y$ divergence.

In dioecious plants of the genus Silene, few pairs of $X$ and $\mathrm{Y}$ linked loci are available as yet, so distinct strata are not detectable, but synonymous site divergence values again differ greatly between different loci, with the highest divergence in regions furthest from the PAR (Nicolas et al, 2005). Similar overall patterns thus evidently evolved independently in phylogenetically very distant animal and plant lineages. Divergence differences may again be partly due to transpositions of genes onto the $\mathrm{Y}$, but so far only a single functional Y-linked gene is known to have arisen in this way, the SlAp3Y gene, which has no X-linked copy, but only an autosomal homologue (Matsunaga et al, 2003). The transposition appears to have occurred soon after the $\mathrm{X}$ and $Y$ chromosomes started to diverge (Matsunaga et al, 2003), but it is unknown whether its initial transfer to the $\mathrm{Y}$ was selectively favoured (it may merely be a part of a larger translocation that was favoured because it carried some other gene(s) with advantages for male functions). Small transpositions cannot explain the observed pattern of divergence along the $\mathrm{X}$ chromosome. It is not yet clear whether inversions have occurred on the Silene X or Y, or both chromosomes, but the data imply multiple evolutionary steps leading to reduced recombination, perhaps even a more-or-less continuous reduction of recombination, and show that reduced recombination can occur over a short time scale, since one gene that is clearly nonrecombining has only $2 \% X-Y$ synonymous site divergence (Filatov et al, 2000). The largest silent site divergence values in Silene, $\sim 25 \%$, are similar to those for the most recent human stratum, 4, or the lowest bird divergence (Lawson-Handley et al, 2004).

Can recombination decrease without chromosome rearrangements, just by spread of recombination modifiers with small effects? Crossing over in the mammalian amelogenin region seems to have been suppressed through several small-scale events (Marais and Galtier, 2003), but these might be small inversions, and other events, such as insertions of transposable elements, are not excluded. Another possibility is that, as it evolved, recombination suppression itself could inhibit recombination in nearby regions of the sex chromosomes. For instance, if recombination at the pseudoautosomal boundary is sufficiently rare, $\mathrm{X}$ and $\mathrm{Y}$ sequences might diverge, perhaps eventually inhibiting recombination and resulting in movement of the boundary (Ellis et al, 1990). However, this seems unlikely since the crossing over rate at the centromeric boundary is very high in the human PAR1 (Lien et al, 2000) and possibly also in mice (Montoya-Burgos et al, 2003).

Whatever the mechanism, loss of $\mathrm{X}-\mathrm{Y}$ recombination has occurred in different taxa, including repeated events 
in different fairly closely related lineages of birds (Ellegren and Carmichael, 2001) and insects (Drosophila and mosquitoes, which have different sex chromosome gene content; Krzywinski et al, 2004). The fact that this evolutionary change occurs repeatedly suggests that recombination suppression between the sex chromosomes was actively favoured by selection, rather than being a consequence of rearrangements such as occasionally occur on all chromosomes. In the amelogenin region, phylogenetic analysis (see Figure 2c) suggests that recombination stopped before the mammalian radiation in the $5^{\prime}$ region, but later in the $3^{\prime}$ region, implying that the recombination suppression leading to the formation of stratum 4 evolved independently in each mammalian order (Iwase et al, 2003).

Despite this evidence suggesting selection for recombination suppression, no direct evidence yet connects this with sexually antagonistic selection. Many Y-linked male function genes are known in humans, and the sequencing of the $\mathrm{Y}$ has shown that two such genes (CDY and DAZ) were transposed from autosomes to the $Y$ (Skaletsky et al, 2003), as has also occurred in Drosophila (Carvalho et al, 2001a,b). To show that this is due to sexually antagonistic selection will require evidence that possession of these male function genes would be deleterious to females. An example of the evolution of suppression of crossing over between sex chromosomes has recently been described in $D$. americana, where one chromosomal inversion is confined to the neo- $X$ chromosomes, and is never found on either the homologous unfused autosome or the neo-Y (McAllister, 2003). Sequence polymorphism studies suggest that the spread of this inversion was driven by natural selection, since closely linked loci show signs of a recent selective sweep. The inversion increases genetic isolation between the neo- $X$ and neo- $Y$ chromosomes, which is incomplete for loci not closely linked to the centromere, since females carrying the neo-Y can be produced when females carrying the unfused chromosome mate with males with the fusion, in populations that are polymorphic for the neo-X chromosomes (McAllister, 2002, 2003).

\section{The time scale of genetic degeneration}

The origins of Drosophila neo-sex chromosomes systems can often be dated by comparisons of related species or species groups, providing a time scale for the degeneration of neo- $Y$ chromosomes. The neo- $Y$ chromosome of D. pseudoobscura and its relatives originated roughly 13 million years ago (Tamura et al, 2004) and is absent from the Old World members of the obscura group; it appears to be almost completely degenerated (Carvalho and Clark, 2005). All the neo-X loci studied now show sexlinked inheritance and apparently full dosage compensation (Lucchesi, 1978; Marín et al, 2000). In contrast, the more recently formed neo-Y of its relative $D$. miranda carries a mixture of missing, degenerate and active loci, with patchy dosage compensation along the neo-sex chromosomes (Steinemann and Steinemann, 1998). In neo-Y genes with no overt signs of degeneration, aminoacid substitution is accelerated, consistent with accumulation of slightly deleterious mutations on this chromosome (Bachtrog, 2003b). The degree of DNA sequence divergence between homologous loci on the neo- $X$ and neo-Y suggests that the fusion that generated the neo-sex chromosomes occurred about one million years ago, about half the $D$. pseudoobscura-D. miranda divergence time. In contrast, $D$. americana neo- $Y$ chromosomes show no evidence for any degeneration, consistent with a much more recent origin and incomplete isolation from the neo-X (Charlesworth et al, 1997). The same appears to be true of the neo-Y of D. albomicans (D Bachtrog, unpublished data), which is so closely related to $D$. nasuta that sequence divergence is minimal ( $\mathrm{Yu}$ et al, $1999 b)$. It is thus clear that a Y chromosome can partially degenerate in fewer than several million generations, as predicted by population genetic models of large nonrecombining genomic regions (Charlesworth and Charlesworth, 2000).

The D. miranda neo-Y also illustrates the tendency of nonrecombining genomic regions to accumulate transposable elements. Several families of elements are in much higher abundance than elsewhere in the genome (Steinemann and Steinemann, 1998), and elements have spread to fixation within the neo-Y chromosome population (Bachtrog, 2003a). As much as 50\% of the DNA of the D. miranda neo-Y may consist of transposable elements (Bachtrog, 2003a), perhaps accounting for its heterochromatic appearance in polytene chromosome preparations (Steinemann and Steinemann, 1998). The Bombyx W chromosome also contains nested retrotransposons, similar to those in maize intergenic regions (Fu and Dooner, 2003), showing that accumulation has been going on for a long time (Abe et al, 2000).

Apart from these well-studied Drosophila examples, the fate of chromosome arms translocated onto sex chromosomes is not well understood. In species where recombination occurs in both sexes, neo- $X$ and $-Y$ chromosomes will cross over, except in the centromeric regions (Davisson and Akeson, 1993; Vieira et al, 2003), and their genetic isolation requires mechanisms suppressing crossing over more widely, like those discussed earlier. Given the evidence for several pairs of formerly autosomal genes on both the $X$ and $Y$ in eutherian mammals (Waters et al, 2001), two fusion events must have occurred; first, the ancestral $\mathrm{X}$ or $\mathrm{Y}$ became fused with an ancestral autosome, and then the free homologue of the formerly autosomal neo-sex chromosome must have fused with the nonfused sex chromosome, perhaps to stabilise segregation in rearrangement heterozygotes (Yu et al, 1999a). There is also evidence for such a dual fusion in D. albomicans (Yu et al, 1999a).

Much less is known about degeneration of Y-linked genes of plants. Sex chromosomes may have evolved independently within several plant genera. The Y copy of the MROS3 gene of Silene latifolia was found to be degenerate (Guttman and Charlesworth, 1998), but as this is a member of a gene family with several other members, one cannot exclude the possibility that this case represents only the normal gene birth and death process of paralogous genes, rather than a phenomenon specific to the $\mathrm{Y}$ chromosome, although there is evidence for low viability of Silene plants lacking an $X$ chromosome (Westergaard, 1958; Ye et al, 1990). Five S. latifolia Ylinked genes with $X$-linked homologues have now been studied, one of which is only a degenerate fragment (Guttman and Charlesworth, 1998), and one is apparently not transcribed (Nicolas et al, 2005), but transcription levels of the others have not yet been compared with their X-linked counterparts. The results from Silene genes 
do not estimate the fraction of degenerate genes. They tell us only that some genes can be maintained on the $\mathrm{Y}$ without becoming pseudogenes, although, even in these, slight degeneration is detectable by detailed comparisons of amino-acid sequence divergence of orthologous $X$ and $\mathrm{Y}$ coding sequences (Nicolas et al, 2005).

\section{Young sex chromosomes}

Data on young sex chromosomes are of great interest, particularly for testing questions such as whether the sex-determining genes are initially on a single chromosome, as the theory outlined above predicts, and also whether accumulation of repetitive sequences is the earliest event, before genes start degenerating. In some fish and plant species, the sex-determining genes have been genetically mapped to small regions of ordinary chromosomes, and some species show no sign of any extended sex chromosomal region, for example, Ficus fulva (Parrish et al, 2004). The best-studied plant case to date is papaya (Liu et al, 2004). There is a small nonrecombining region of roughly $4.4 \mathrm{Mb}$, only about $10 \%$ of chromosome 1 (but probably containing 100-200 genes, based on gene densities in other papaya genome regions). AFLP marker densities in the male-specific region are, however, much higher than for the rest of chromosome 1 (225 markers cosegregate with sex, while the rest of the chromosome, at least $41 \mathrm{Mb}$, yielded only 117 markers in the same screen). This strongly suggests divergence between 'Y-like' and ' $X$-like' homologous regions. However, it is not yet clear whether this represents divergence in genes - it might represent accumulated differences in intergenic regions since the 'Y-like' and 'X-like' homologous regions ceased recombining. The male-specific region is indeed enriched with repetitive sequences, compared with other genome regions, like other Y chromosomes (Skaletsky et al, 2003)

The sex-determining region in three-spine sticklebacks, Gasterosteus aculeatus, is similar, although only about $250 \mathrm{~kb}$ of this region are well characterised (Peichel et al, 2004). Crossing over in males is suppressed over a wide region near a sex-determining factor located at one end of the proto- $X$ and proto- $Y$ chromosomes (linkage group 19): two markers separated by 16-20 cM in females on the proto- $X$ chromosome are only $1.5-3.5 \mathrm{cM}$ apart in males. Repetitive sequences have accumulated in the male-specific region, including transposable elementderived sequences, reducing sequence similarity between intergenic sequences of the proto- $X$ and proto- $Y$ to below $64 \%$.

The related stickleback species $G$. wheatlandi appears to have a different sex-determining system, with cytologically distinct X and Y chromosomes (Peichel et al, 2004). The G. aculeatus sex determination region may thus have evolved fairly recently, as a result of a male determining gene having been transposed onto linkage group 19 from elsewhere. Such an origin of the proto-Y chromosome is strongly supported in the case of the medaka, O. latipes, another fish species that has been studied in detail at the molecular level. As in the stickleback, crossing over is strongly reduced near the sex-determining region (Kondo et al, 2001). Part of the male-specific genome region containing the male determining factor has now been characterised in detail. It contains a gene, $d m r t 1 b Y$, which is required for male development (Matsuda et al, 2002;
Nanda et al, 2002). This is related to DMRT1 of mammals (which plays a major role in controlling sexual phenotype), and to genes implicated in the sex determination pathways of other animals, including Caenorhabditis elegans and D. melanogaster, and reptiles with environmental sex determination (Zarkower, 2002). The malespecific region of the medaka proto- $Y$ has accumulated repetitive sequences, and other genes in the region seem to have become nonfunctional (Nanda et al, 2002). The region is only about $260 \mathrm{~kb}$ in length, representing about $1 \%$ of the length of the chromosome, with no homologous region present on the proto- $X$ chromosome. Dmrt1by seems to have originated by a recent duplication (estimated to have occurred 4-10 MYA) of an autosomal gene, $d m r t 1 a$, which is present only in close relatives of the medaka (Kondo et al, 2004). Why this duplication was established, and why crossing over is suppressed close to dmrt1bY, remain unknown.

\section{Are plant and fish sex chromosomes really young?}

In studying plant and fish sex chromosomes, an important issue is to test whether they are truly primitive, and are just starting on the path that may ultimately lead to heteromorphic sex chromosome evolution, rather than being derived from pre-existing sex chromosomes. Their ages can be estimated, subject to the uncertainty of molecular clock dating, if reliably alignable sequence data (from coding sequences) are available to estimate $\mathrm{X}-\mathrm{Y}$ divergence. To compare patterns of $X$ and $Y$ sequence divergence, including testing whether the $X$ and $Y$ mutation rates differ ( $\mathrm{Li}$ and Makova, 2002), and to understand the history of chromosome rearrangements, data from outgroup species with orthologous sequences are also needed (Filatov and Charlesworth, 2002).

The scattered distribution of dioecy in flowering plants suggests at first sight that sex chromosomes evolved within those families where they are found, but it is difficult to rule out an ancient origin of dioecy, with secondary reversion to hermaphroditism within many plant families. Insect-pollinated plants are probably vulnerable to unreliable pollination, which can strongly select for hermaphrodite function, and cases of reversion are known (Huff and Wu, 1992; Rieseberg et al, 1992; Pannell, 1997); not surprisingly, males of many plant species have some ability to set fruits (some species have 'inconstant males' where this regularly occurs; Darwin, 1877; Burrows, 1960). Consistent with reversion to hermaphroditism occurring regularly, dioecious clades of plants generally have lower species richness than their sister clades (Heilbuth, 2000).

It will therefore be important to combine data from taxa with putatively young sex chromosome systems with phylogenetic analysis, in order to place the estimated origins of sex chromosomes in the context of the history of the taxa. This will require comparing divergence from extant hermaphrodite relatives with $X-$ $Y$ gene divergence, to test whether dioecy really evolved from a common ancestor with the hermaphrodite species, or might have originated earlier. Obtaining outgroups may pose a problem for evolutionary studies in fish such as sticklebacks, where related species are also dioecious, and plants such as papaya, where most species in the entire (small) family are dioecious. If 
dioecy evolved in an ancestor, rather than within the genus, it may be difficult to find outgroup species at divergence levels suitable for analyses. On the other hand, an ancient origin would be interesting, as the small size of the nonrecombining region (Liu et al, 2004) would then suggest that suppressed recombination can remain limited in extent, perhaps because sexually antagonistic genes have not accumulated to any considerable extent; degeneration of the entire $\mathrm{Y}$ chromosome may thus not be inevitable. This possibility is consistent with the evolutionary models, which do not imply that proto- $X$ and proto-Y chromosomes will necessarily evolve complete crossover suppression and $\mathrm{Y}$ chromosome degeneration. It will thus be very interesting when $X-Y$ divergence data become available for papaya coding sequences, making possible comparisons of the ages of the papaya and Silene male-specific regions.

\section{Conclusions}

We have emphasised that sex chromosomes can evolve in several different ways, and that recombination suppression is favoured when genes are involved that interact with sex with respect to their effects on fitness There are two situations in which such interactions are likely: (i) in the initial stages of evolution of dioecy, and (ii) after dioecy has been established, including situations when a sex-determining gene has moved to a new genome location, or control of sex determination has been usurped by a new sex-determining gene. The models outlined above predict that the $Y$ will be subject to several successive changes driven by selection (Figure 1). Increasing knowledge of the detailed anatomy and gene content of sex chromosomes and male-specific regions of chromosomes should allow these ideas to be tested.

More studies of $\mathrm{X}$ - and Y-linked genes, and more detailed genetic maps of sex chromosomes in a range of species are therefore needed. Transposable element accumulation is probably an early effect of restricted recombination, so one of the first changes affecting sex chromosomes may be increased DNA content. Plant $Y$ chromosomes are often larger than the $\mathrm{X}$ chromosomes (Parker, 1990), consistent with recent origins; the large chromosome size does not imply absence of genetic degeneration, nor a high expressed gene content. On the other hand, accumulation of repetitive sequences such as transposable elements on the $\mathrm{Y}$ does not necessarily imply degeneration. It may merely be a sign that recombination is rare; accumulation also occurs in nonrecombining regions of the Drosophila genome (Charlesworth et al, 1994; Bartolomé and Maside, 2004). The reasons for transposable element build-up probably involve several processes. Elements that would normally be eliminated by ectopic exchange may fail to be eliminated from the neo-Y, due to lack of recombination. The efficacy of selective removal will also be reduced because of low recombination rates (Charlesworth et al, 1994). Genetic degeneration may be partly driven by this accumulation, or may occur independently later on. In the $D$. miranda neo-Y, transposable elements appear to be inserted either into introns or intergenic sequences, and hence do not directly disrupt gene function (although one insertion causes downregulation of a gene; Steinemann and Steinemann, 1992, 1998; Bachtrog, 2003a). It is possible that a build-up of transposable elements on a proto-Y or neo-Y could reduce gene expression on these chromosomes, through chromatin modification due to dsRNA interference, which is known to be associated with the presence of transposable elements (Lippman and Martienssen, 2004). This could affect the selective constraints on neo-Y chromosomal genes, allowing faster degeneration than would happen in sex chromosome systems evolving de novo. Given the evidence for accumulation of transposable elements in the papaya and stickleback male-specific regions, it will be interesting in the future to test whether they are genetically degenerate. This will require laborious work to isolate $X$ linked genes, and then to test whether the $Y$ chromosome carries functional or degenerated copies.

There is no substitute for ascertainment of expressed sex-linked genes, and detailed molecular evolutionary studies. Most of the few plant sex-linked genes that have been studied so far were obtained from cDNA probed with Y chromosome sequences (Nicolas et al, 2005), creating a bias towards transcribed Y-linked genes, and thus possibly towards nondegenerate genes. At present, the results from Silene genes can tell us only that some genes can be maintained on the $\mathrm{Y}$ without becoming pseudogenes. The only unbiased way to determine the fraction of degenerate loci is to ascertain X-linked genes. Once such genes are available, it will be possible to compare the time scale of transposable element accumulation with that of changes in coding sequences. It is clear that, over a time scale of only about one million years, about $30 \%$ of the genes so far studied on the D. miranda neo-Y are degenerate (with major lesions, or deleted; Steinemann and Steinemann, 1998; Bachtrog, 2003b). However, in this case, a whole autosomal chromosome arm containing over 2000 genes is involved. This creates a large opportunity for Hill-Robertson interference processes that impede the efficacy of selection, detectable from the large reduction in effective population size for the neo-Y (Bachtrog and Charlesworth, 2002).

In recently evolved sex chromosomes, degeneration is probably much slower than in neo-Y chromosomes. There is less opportunity for selection to cause reduced effective population sizes of $\mathrm{Y}$ - or $\mathrm{W}$-specific genes, since only loci within the sex-specific region are probably relevant. However, the Silene $\mathrm{Y}$ chromosome has low diversity, suggesting a detectable reduction in its effective size (Filatov et al, 2000; Filatov et al, 2001). Given that the stickleback and papaya male-specific regions have accumulated repetitive DNA, they may have been isolated from recombination with $X$-linked homologues for quite long times. Divergence data for synonymous sites within coding sequences should help to estimate the time scales.

Sequences from sex-linked loci will also allow accurate measurements of gene expression, important for testing whether Y- or W- linked genes lose function gradually, which would be consistent with hitch-hiking processes increasing frequencies of maladapted alleles, rather than major mutations causing loss of functions. The relationship between gene degeneration and dosage compensation cannot be studied until X-linked genes with expression in both sexes are found.

Knowledge of the $X$ and $Y$ gene content will also make it possible to map the sex chromosomes of nonmodel species of evolutionary interest. A detailed $\mathrm{X}$ recombina- 
tion map should allow detection of discontinuities in $\mathrm{X}-$ $Y$ divergence, allowing the chromosome rearrangement hypothesis to be tested against more gradual recombination suppression. Deletion maps of the $\mathrm{Y}$ or $\mathrm{W}$ should illuminate the history of any rearrangements, and indicate the locations of active loci, including the sexdetermining genes. Genetic maps without physical map data cannot distinguish between large nonrecombining regions and smaller regions in which insertions of repetitive sequences and subsequent insertion/deletion events have been accumulating over long evolutionary times. Large numbers of anonymous markers, such as ALFP, can thus readily be found, not only when the male-specific region of a sex chromosome is physically large (and the pseudoautosomal region relatively small) but also if $X-Y$ divergence is high, leading to disproportionately many marker differences between them. Conversely, we can infer that species with few completely Y-linked markers must have small nonrecombining regions, provided that large numbers of markers are scored. Mapping sequence variants in functional genes, including allozyme markers, will also be very helpful. Sex-specific markers can be found even when $X-Y$ divergence is slight (e.g. the completely Y-linked $S$. latifolia SIY1 marker, whose synonymous site divergence is only a few per cent Filatov et al, 2000). When many pseudoautosomal genes are found, this suggests a large PAR, as may be the state in many fish (Marshall et al, 2004).

If Y-linked genes are incessantly evolving to improve male function and compete with other male genotypes, the changes may drive the evolution of recombination suppression, and thus recombination modifiers may also spread, some of which may themselves be on the Y (for instance, inversions). In established systems with a largely nonrecombining $\mathrm{Y}$, these selection processes might be detectable, because chromosome-wide selective sweeps would occur, which could lower diversity of Ylinked genes, in combination with other hitch-hiking processes. If this contributes to the observed low Ylinked gene diversity, as suggested by data on the $D$. miranda neo-Y chromosome (Bachtrog, 2004), variants should be at low frequencies, which can be detected by tests based on variant frequencies such as Tajima's test (Tajima, 1989). However, if selection events are too frequent, or hitch-hiking processes reduce diversity too greatly, there might be too little diversity to apply these tests unless very long sequences are obtained.

Directly detecting adaptive changes on the $\mathrm{Y}$ and sexually antagonistic selection is a major task for the future. Selection could potentially be detected from accelerated amino-acid evolution at the loci involved, especially if several branches of a phylogenetic tree are available, so that methods such as PAML for directly detecting selection on the loci affected can be used (Yang and Bielawski, 2000), but finding good candidate loci that are potential targets of selection is very difficult. Perhaps the most favourable situation for obtaining evidence for sexually antagonistic selection is in species whose sex chromosome systems evolved fairly recently, and are still experiencing the selective changes, such as Silene or fish, or where sex differences in allele frequencies of partially sex-linked genes can suggest such selection (Fisher, 1931; Marshall et al, 2004). The presence of genes for male characters on the $Y$, but not the $X$, also indirectly suggests that antagonistic selection must have acted. When there has been a recent transposition of an autosomal gene onto the $\mathrm{Y}$ chromosome, if sufficient intraspecies variability is available, tests for selection based on comparisons of polymorphism and divergence (McDonald and Kreitman, 1991) could be used to ask whether the locus has experienced a burst of adaptive evolution. Transgenic experiments could also be used to test the genes' effects in females.

\section{References}

Abe H, Ohbayashi F, Shimada T, Sugasaki T, Kawai S, Mita K et al (2000). Molecular structure of a novel gypsy-Ty3-like retrotransposon (Kabuki) and nested retrotransposable elements on the $\mathrm{W}$ chromosome of the silkworm Bombyx mori. Mol Gen Genet 263: 916-924.

Ashburner M 1989)). Drosophila. A Laboratory Handbook. Cold Spring Harbor Press: Cold Spring Harbor, NY.

Bachtrog D (2003a). Accumulation of Spock and Worf, two novel non-LTR retrotransposons, on the neo-Y chromosome of Drosophila miranda. Mol Biol Evol 20: 173-181.

Bachtrog D (2003b). Protein evolution and codon usage bias on the neo-sex chromosomes of Drosophila miranda. Genetics 165: $1221-1232$

Bachtrog D (2004). Evidence that positive selection drives Y-chromosome degeneration in Drosophila miranda. Nat Genet 36: 518-522.

Bachtrog D, Charlesworth B (2002). Reduced adaptation of a non-recombining neo-Y chromosome. Nature 416: 323-326.

Barlow BA, Wiens D (1976). Translocation heterozygosity and sex ratio in Viscum fischeri. Heredity 37: 27-40.

Barrett SCH (1992). Heterostylous genetic polymorphisms: model systems for evolutionary analysis. In: Omary A (ed) Evolution and Function of Heterostyly. Springer-Verlag: Heidelberg, pp 1-29.

Bartolomé C, Maside X (2004). The lack of recombination drives the fixation of transposable elements on the fourth chromosome of Drosophila melanogaster. Genet Res 83: 91-100.

Brooks LD (1988). The evolution of recombination rates. In: Michod RE, Levin BR (eds) The Evolution of Sex Sinauer. Sunderland: Massachusetts pp 87-105.

Bull JJ (1983). Evolution of Sex Determining Mechanisms. Benjamin/Cummings: Menlo Park, CA.

Burrows CJ (1960). Studies in Pimelea. I. The breeding system. Trans R Soc New Zealand 88: 29-45.

Carvalho AB (2002). Origin and evolution of the Drosophila $Y$ chromosome. Curr Opin Genet Dev 12: 664-668.

Carvalho AB, Clark AG (2005). Y chromosome of D. pseudoobscura is not homologous to the ancestral Drosophila Y. Science 307: 108-110.

Carvalho AB, Dobo BA, Vibranovski MD, Clark AG (2001b). Identification of five new genes on the $\mathrm{Y}$ chromosome of Drosophila melanogaster. Proc Natl Acad Sci USA 98: 1322513230.

Carvalho AB, Lazzaro BP, Clark AG (2001a). Y chromosomal fertility factors kl-2 and kl-3 of Drosophila melanogaster encode dynein heavy chain polypeptides. Proc Natl Acad Sci USA 97: 13239-13244.

Charchar FJ, Svartman M, El-Mogharbel N, Ventura M, Kirby P, Matarazzo MR et al (2003). Complex events in the evolution of the human pseudoautosomal region 2 (PAR2). Genome Res 13: 281-286.

Charlesworth B (1978). Model for evolution of $\mathrm{Y}$ chromosomes and dosage compensation. Proc Natl Acad Sci USA 75: $5618-5622$.

Charlesworth B (1996). The evolution of chromosomal sex determination and dosage compensation. Curr Biol 6: 149-162. 
Charlesworth B (2002). The evolution of chromosomal sex determination. In: Short R (ed) The Genetics and Biology of Sex Determination. John Wiley: Chichester, UK. pp 207-219.

Charlesworth B, Charlesworth D (1978). A model for the evolution of dioecy and gynodioecy. Am Nat 112: 975-997.

Charlesworth B, Charlesworth D (2000). The degeneration of Y chromosomes. Philos Trans R Soc Lond Ser B 355: 15631572 .

Charlesworth B, Hartl DL (1978). Population-dynamics of the segregation distorter polymorphism of Drosophila melanogaster. Genetics 89: 171-192.

Charlesworth B, Charlesworth D, Yu A, Hnilicka J (1997). Evidence for lack of degeneration of allozyme loci on the fourth chromosomes of Drosophila americana. Genetics 145: 989-1002.

Charlesworth B, Sniegowski P, Stephan W (1994). The evolutionary dynamics of repetitive DNA in eukaryotes. Nature 371: 215-220.

Charlesworth D, Charlesworth B (1980). Sex differences in fitness and selection for centric fusions between sexchromosomes and autosomes. Genet Res 35: 205-214.

Darwin CR (1877). The Different Forms of Flowers on Plants of the Same Species. John Murray, London.

Davisson MT, Akeson EC (1993). Recombination suppression by heterozygous Robertsonian fusions in the mouse. Genetics 133: 649-667.

Ellegren H, Carmichael A (2001). Multiple and independent cessation of recombination between avian sex chromosomes. Genetics 158: 325-331.

Ellis N, Yen P, Neiswanger K, Shapiro LJ, Goodfellow P (1990). Evolution of the pseudoautosomal boundary in Old-world monkeys and great apes. Nature 63: 977-986.

Ferris PI, Armbrust EV, Goodenough U (2002). Genetic structure of the mating-type locus of Chlamydomonas reinhardtii. Genetics 160: 181-200.

Filatov DA, Charlesworth D (2002). Substitution rates in the $\mathrm{X}$ - and Y-linked genes of the plants, Silene latifolia and $S$. dioica. Mol Biol Evol 19: 898-907.

Filatov DA, Laporte V, Vitte C, Charlesworth D (2001). DNA diversity in sex linked and autosomal genes of the plant species Silene latifolia and S. dioica. Mol Biol Evol 18: 1442-1454.

Filatov DA, Monéger F, Negrutiu I, Charlesworth D (2000). Evolution of a plant Y-chromosome: variability in a Y-linked gene of Silene latifolia. Nature 404: 388-390.

Fisher RA (1931). The evolution of dominance. Biol Rev 6: 345-368.

Fraser JA, Heitman J (2004). Evolution of fungal sex chromosomes. Mol Microbiol 51: 299.

$\mathrm{Fu} \mathrm{H}$, Dooner HK (2003). Intraspecific violation of genetic colinearity and its implications in maize. Proc Natl Acad Sci USA 99: 9573-9578.

Guttman DS, Charlesworth D (1998). An X-linked gene has a degenerate Y-linked homologue in the dioecious plant Silene latifolia. Nature 393: 263-266.

Heilbuth JC (2000). Lower species richness in dioecious clades. Am Nat 156: 221-241.

Huff DR, Wu L (1992). Distribution and inheritance of inconstant sex forms in natural populations of dioecious buffalograss (Buchloe dactyloides). Am J Bot 79: 207-215

Ishizaki K, Shimizu-Ueda Y, Okada S, Yamamoto M, Fujisawa M, Yamato KT et al (2002). Multicopy genes uniquely amplified in the $\mathrm{Y}$ chromosome-specific repeats of the liverwort Marchantia polymorpha. Nucleic Acids Res 30: 4675-4681.

Iwase M, Satta Y, Hirai Y, Hirai H, Imai H, Takahata N (2003). The amelogenin loci span an ancient pseudoautosomal boundary in diverse mammalian species. Proc Natl Acad Sci USA 100: 5258-5263.

Janzen FJ, Krenz JG (2004). Phylogenetics: which was first, TSD or GSD? In: Lance NVAV (ed) Temperature Dependent Sex
Determination in Vertebrates. Smithsonian Books: Washington, DC. pp 121-130.

Kondo M, Nagao E, Mitani H, Shima A (2001). Differences in recombination frequencies during male and female meioses of the sex chromosomes of the medaka, Orzyias latipes. Genet Res 78: 23-30.

Kondo M, Nanda I, Hornung U, Schmid M, Schartl M (2004). Evolutionary origin of the medaka Y-chromosome. Curr Biol 14: 1664-1669.

Krzywinski J, Nusskern DR, Kern MK, Besansky NJ (2004). Isolation and characterization of $\mathrm{Y}$ chromosome sequences from the African malaria mosquito Anopheles gambiae. Genetics 166: 1291-1302.

Kurian V, Richards AJ (1997). A new recombinant in the heteromorphy ' $\mathrm{S}$ ' supergene in Primula. Heredity 78: 383-390.

Lahn BT, Page DC (1999). Four evolutionary strata on the human X chromosome. Science 286: 964-967.

Lahn BT, Pearson NM, Jegalian K (2001). The human Y chromosome, in the light of evolution. Nat Rev Genet 2: 207-216.

Lawson-Handley LJ, Ceplitis H, Ellegren H (2004). Evolutionary strata on the chicken $\mathrm{Z}$ chromosome: implications for sex chromosome evolution. Genetics 167: 367-376.

Li WH, Makova KD (2002). Male-driven evolution. Curr Opin Genet Dev 12: 650-656.

Lien S, Szyda J, Schechinger B, Rappold G, Arnheim N (2000). Evidence for heterogeneity in recombination in the human pseudoautosomal region: high resolution analysis by sperm typing and radiation-hybrid mapping. Am J Hum Genet 66 : 557-566.

Lindholm A, Breden F (2002). Sex chromosomes and sexual selection in poeciliid fishes. Am Nat 160: S214-S224.

Lippman Z, Martienssen R (2004). The role of RNA interference in heterochromatic silencing. Nature 431: 364-370.

Liu Z, Moore PH, Ma H, Ackerman CM, Ragiba M, Pearl HM et al (2004). A primitive $Y$ chromosome in papaya marks the beginning of sex chromosome evolution. Nature 427: 348-352.

Lucchesi JC (1978). Gene dosage compensation and the evolution of sex chromosomes. Science 202: 711-716.

Marais G, Galtier N (2003). Sex chromosomes: how X-Y recombination stops. Curr Biol 13: R641-R643.

Marín IM, Siegal ML, Baker BS (2000). The evolution of dosage compensation mechanisms. Bioessays 22: 1106-1114.

Marshall AR, Knudsen KL, Allendorf FW (2004). Linkage disequilibrium between the pseudoautosomal PEPB-1 locus and the sex-determining region of chinook salmon. Heredity 93: 85-97.

Mather K, Winton DD (1941). Adaptation and counter-adaptation of the breeding system in Primula. Ann Bot ns 5: 297-311.

Matsuda M, Nagahama Y, Shinomiya A, Sato T, Matsuda C, Kobayashi $\mathrm{T}$ et al (2002). DMY is a Y-specific DM-domain gene required for male development in the medaka fish. Nature 417: 559-563.

Matsunaga S, Isono E, Kejnovsky E, Vyskot B, Kawano S, Charlesworth D (2003). Duplicative transfer of a MADS box gene to a plant Y chromosome. Mol Biol Evol 20: 1062-1069.

Maynard Smith J (1978). The Evolution of Sex. Cambridge University Press: Cambridge.

McAllister BF (2002). Chromosomal and allelic variation in Drosophila americana: selective maintenance of a chromosomal cline. Genome 45: 13-21.

McAllister BF (2003). Sequence differentiation associated with an inversion on the neo- $X$ chromosome of Drosophila americana. Genetics 165: 1317-1328.

McDonald JH, Kreitman M (1991). Accelerated protein evolution at the Adh locus in Drosophila. Nature 351: 652-654.

Montoya-Burgos JI, Boursot P, Galtier N (2003). Recombination explains isochores in mammalian genomes. Trend Genet 19 128-130.

Nanda I, Kondo M, Hornung U, Asakawa S, Winkler C, Shimizu A et al (2002). A duplicated copy of DMRT1 in the 
sex-determining region of the $\mathrm{Y}$ chromosome of the medaka, Oryzias latipes. Proc Natl Acad Sci USA 99: 11778-11783.

Nei M (1969). Linkage modification and sex difference in recombination. Genetics 63: 681-699.

Nei M (1970). Accumulation of nonfunctional genes on sheltered chromosomes. Am Nat 104: 311-322.

Nicolas M, Marais G, Hykelova V, Janousek B, Laporte V, Vyskot B et al (2005). A gradual process of recombination restriction in the evolutionary history of the sex chromosomes in dioecious plants. PLoS Biol 3: 47-56.

Nokkala S, Grozeva S, Kuznetsova V, Maryanska-Nadachowska A (2003). The origin of the achiasmatic XY sex chromosome system in Cacopsylla peregrina (Frst.) (Psylloidea, Homoptera). Genetica 119: 327-332.

Orr HA, Kim Y (1998). An adaptive hypothesis for the evolution of the Y chromosome. Genetics 150: 1693-1698.

Pannell J (1997). Variation in sex ratios and sex allocation in androdioecious Mercurialis annua. J Ecol 85: 57-59.

Parker JS (1990). Sex-chromosome and sex differentiation in flowering plants. Chromosomes Today 10: 187-198.

Parrish TL, Koelewijn HP, Dijk PJv (2004). Identification of a male-specific AFLP marker in a functionally dioecious fig, Ficus fulva Reinw. ex Bl. (Moraceae). Sex Plant Reprod 17: $17-22$.

Pecon-Slattery J, Sanner-Wachter L, O'Brien SJ (2000). Novel gene conversion between $\mathrm{X}-\mathrm{Y}$ homologues located in the nonrecombining region of the $\mathrm{Y}$ chromosome in Felidae (Mammalia). Proc Natl Acad Sci USA 97: 5307-5312.

Peichel CL, Ross JA, Matson CK, Dickson M, Grimwood J, Schmutz J et al (2004). The master sex-determination locus in threespine sticklebacks is on a nascent $\mathrm{Y}$ chromosome. Curr Biol 14: 1416-1424.

Perry J, Ashworth A (1999). Evolutionary rate of a gene affected by chromosomal position. Curr Biol 9: 987-989.

Rejón CR, Jamilena M, Ramos MG, Parker JS, Rejón MR (1994). Cytogenetic and molecular analysis of the multiple sexchromosome system of Rumex acetosa. Heredity 72: 209-215.

Rens W, Grützner F, O'Brien PCM, Fairclough H, Jones RC, Graves JAM et al (2004). Resolution and evolution of the duck-billed platypus karyotype with an X1Y1X2Y2X3Y3X4Y4X5Y5 male sex chromosome constitution. Proc Natl Acad Sci USA 101: 16257-16261.

Rice WR (1987a). The accumulation of sexually antagonistic genes as a selective agent promoting the evolution of reduced recombination between primitive sex-chromosomes. Evolution 41: 911-914.

Rice WR (1987b). Genetic hitch-hiking and the evolution of reduced genetic activity of the $\mathrm{Y}$ sex chromosome. Genetics 116: $161-167$.

Rice WR (1996). Evolution of the Y sex chromosome in animals. Biosciences 46: 331-343.

Rieseberg LH, Hanson MA, Philbrick CT (1992). Androdioecy is derived from dioecy in Datiscaceae: evidence from restriction site mapping of PCR amplified chloroplast DNA. Syst Bot 17: 324-336.

Ross MT, Grafham DV, Coffey AJ, Scherer S, McLay K, Muzny D et al (2005). The DNA sequence of the human X chromosome. Nature 434: 325-337.

Rozen S, Skaletsky H, Marszalek JD, Minx PJ, Cordum HS, Waterston RH et al (2003). Abundant gene conversion between arms of palindromes in human and ape $\mathrm{Y}$ chromosomes. Nature 423: 873-876.

Shearman DCA (2002). The evolution of sex determination systems in Dipteran insects other than Drosophila. Genetica 116: $25-43$.
Skaletsky H, Kuroda-Kawaguchi T, Minx PJ, Cordum HS, Hillier L, Brown LG et al (2003). The male-specific region of the human $\mathrm{Y}$ chromosome is a mosaic of discrete sequence classes. Nature 423: 825-837.

Steinemann M, Steinemann S (1992). Degenerating Y chromosome of Drosophila miranda: a trap for retrotransposons. Proc Natl Acad Sci USA 89: 7591-7595.

Steinemann M, Steinemann S (1998). Enigma of Y chromosome degeneration: neo-Y and neo-X chromosomes of Drosophila miranda a model for sex chromosome evolution. Genetica 102/ 103: 409-420.

Stone JL (2004). Sheltered load associated with S-alleles in Solanum carolinense. Heredity 92: 335-342.

Syren RM, Luykx P (1977). Permanent segmental interchange comples in the termite Incisitermes schwarzi. Nature 266: 167168.

Tajima F (1989). Statistical method for testing the neutral mutation hypothesis. Genetics 123: 585-595.

Takebayashi N, Newbigin E, Uyenoyama MK (2004). Maximum-likelihood estimation of rates of recombination within mating-type regions. Genetics 167: 2097-2109.

Tamura M, Subramanian S, Kumar S (2004). Tempral patterns of fruit fly (Drosophila) evolution revealed by mutation clocks. Mol Biol Evol 21: 36-44.

Traut W, Willhoeft U (1990). A jumping sex determining factor in the fly Megaselia scalaris. Chromosoma (Berl) 99: 407-412.

Traut W, Wollert B (1998). An X/Y DNA segment from an early stage of sex chromosome differentiation in the fly Megaselia scalaris. Genome 41: 289-294.

Veyrunes F, Catalan J, Sicard B, Robinson TJ, Duplantier JM, Granjon L et al (2004). Autosome and sex chromosome diversity among the African pygmy mice, subgenus Nannomys (murinae; Mus). Chromosome Res 12: 369-382.

Vieira CP, Coelho PA, Vieira J (2003). Inferences on the history of the Drosophila americana polymorphic $\mathrm{X} / 4$ fusion from patterns of polymophism at the X-linked paralytic and elav genes. Genetics 164: 1459-1469.

Waters PD, Duffy B, Frost CJ, Delbridge ML, Graves JAM (2001). The human Y chromosome derives largely from a single autosomal region added to the sex chromosomes 80-130 million years ago. Cytogenet Cell Genet 92: $74-79$.

Westergaard M (1958). The mechanism of sex determination in dioecious plants. Adv Genet 9: 217-281.

Willhoeft U, Traut W (1990). Molecular differentiation of the homomorphic sex chromosomes in Megaselia scalaris (Diptera) detected by random DNA probes. Chromosoma (Berl) 99: 237-242.

Yang ZH, Bielawski JP (2000). Statistical methods for detecting molecular adaptation. Trends Ecol Evol 15: 496-503.

Ye D, Installé P, Ciuperescu C, Veuskens J, Wu Y, Salesses G et al (1990). Sex determination in the dioecious Melandrium. I. First lessons from androgenic haploids. Sex Plant Reprod 3: 179-186.

Yu YC, Lin FJ, Chang HY (1999a). Stepwise chromosome evolution in Drosophila albomicans. Heredity 83: 39-45.

Yu H, Wang W, Fang S, Zhang Y-P, Lin F-J, Geng Z-C (1999b). Phylogeny and evolution of the Drosophila nasuta subgroup based on mitochondrial ND4 and ND4L gene sequences. Mol Phylogenet Evol 13: 556.

Zarkower D (2002). Invertebrates may not be so different after all. In: Chadwick D, Goode J (eds) The Genetics and Biology of Sex Determination. John Wiley: Chichester, UK. pp 115-135. 